\title{
Intervención del profesional de enfermería durante la contingencia de influenza A H1N1, en el Instituto Nacional de Enfermedades Respiratorias
}

\author{
Intervention of the professional nursing during \\ the contingency of influenza AH1N1 in \\ the National Institute of respiratory diseases
}

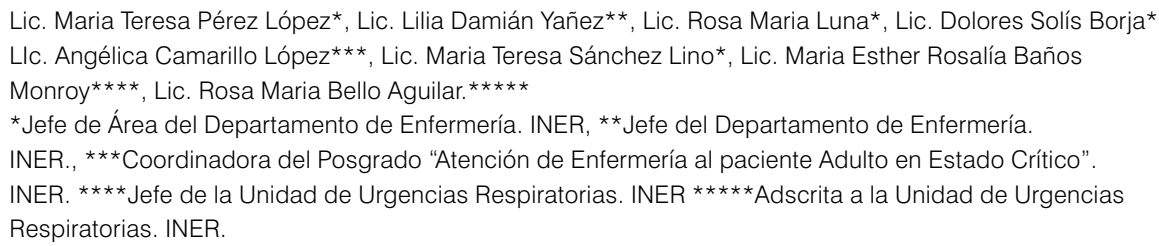

\section{Resumen}

El INER es una institución normativa en las afecciones respiratorias, quien a lo largo de su historia a enfrentado desafíos ante situaciones emergentes de salud, como lo son la tuberculosis y el $\mathrm{VIH}$. Actualmente afronta un nuevo reto de salud respiratoria, la epidemia de influenza $A$ H1N1, la cual se presento de forma inesperada en México en la primavera del 2009, provocando la implementación de medidas sanitarias urgentes, al rebasase la capacidad de atención médica en el instituto. Por ello, fue necesario organizar todos sus recursos humanos, físicos y materiales, Instaurar el Comité de Influenza Institucional y en apoyo y coordinación con el Comité de Control de Infecciones, tomar decisiones urgentes enfocadas a la prevención, manejo y control de la enfermedad. Se Implemento el Triage en los servicios de concentración para la atención integral del paciente con sospecha y/o confirmación de influenza (Consulta Urgencias, Urgencias Observación, Unidades de Terapia Intensiva e Intermedia y clínico 5).

Con todo ello y gracias a la intervención y el trabajo en equipo que realizó el profesional de enfermería del Instituto, se logró controlar la propagación de la epidemia y una vez más lograr conciencia en el personal de salud (médicos y enfermeras), para afrontar de manera exitosa, nuevos retos en la salud respiratoria 


\section{Abstract}

INER is a respiratory-illness treating institution which has faced diverse health challenges including tuberculosis and $\mathrm{VIH}$; yet, at present, it is facing a new one: the unexpected 2009 A H1N1 influenza outbreak in Mexico. This massive incident provoked the implementation of urgent sanitary measurements including: the organization of human, physical and material resources; the establishment of-Infection Committee coordinated measurement towards illness prevention, control and management; and the establishment of the TRIAGE on concentration services towards patients with confirmed or dubious influenza (Urgencies, Consult, Urgencies Observation, Intensive Therapy Unit, Intermediate Therapy Unit and Clinical 5).

Thanks to these measurements and the INER's nursing personnel team work, we were able to control the outbreak propagation, but also to create consciousness among doctors and nurses to successfully face the new respiratory-illnesses challenges.

Key words: AH1N1: Influenza; Nursing care

\section{INTRODUCCIÓN}

El Instituto Nacional de Enfermedades Respiratorias a lo largo de su historia a vivido y superado los retos de atención a la salud tales como: (tuberculosis, fibrosis pulmonar, enfermedades infecciosas del aparato respiratorio y $\mathrm{VIH}$ ), siendo los son padecimientos que se presentan con mayor frecuencia, por ello los médicos, enfermeras y los trabajadores del INER hemos adquirido una conciencia social que nos permite entender la enfermedad con mayor objetividad y abordarla con mayor compromiso. Durante más de 75 años, nuestro instituto ha respondido satisfactoriamente a la tarea que le fue encomendada, la lucha contra las enfermedades respiratorias. ${ }^{1}$

Actualmente el Instituto atraviesa por una contingencia epidemiológica por la Influenza denominada AH1N1, Io que ha generado una creciente demanda de consultas médicas, rebasando la capacidad del servicio de urgencias y hospitalización de pacientes con sospecha de esta enfermedad respiratoria. La emergencia de salud generó el fortalecimiento de medidas sanitarias para mitigar la propagación de la transmisión, siendo estas disposiciones de gran ventaja para manejo, control y prevención de la Influenza A, H1N1 en el INER.

La influenza es una enfermedad viral que afecta anualmente a, aproximadamente 500 millones de personas en el mundo, su presentación es más frecuente durante los meses de invierno y predomina en los lugares donde el clima es árido y frío. El virus de la influenza se propaga rápidamente en todo el mundo de persona a persona dando como resultado epidemias estaciónales.

Sin embargo en el 2009, el comportamiento del virus cambió; reflejándose en las estadísticas, manifestando un aumento en la frecuencia de número de casos en los meses de marzo y abril. Además los pacientes que fueron valorados dentro del Instituto presentaron un cuadro clínico aún más severo de lo observado en años previos.

La influenza es una enfermedad respiratoria viral, contagiosa y aguda, cuyas manifestaciones características son fiebre, cefalea, mialgia, postración, coriza, dolor de garganta y tos. Los síntomas y los signos difieren en función de la edad de las personas infectadas. Las hospitalizaciones y las defunciones se dan principalmente en grupos de alto riesgo: niños menores de dos años, ancianos y enfermos crónicos. ${ }^{2}$

Durante el brote de influenza se observó un aumento en la tasa de morbilidad por influenza en escolares y adultos jóvenes, es decir afectó a un grupo considerado como no vulnerable al virus. Los síntomas que se observaron con mayor frecuencia en la población pediátrica fueron fiebre alta, dificultad respiratoria, rechazo a la vía oral, irritabilidad y/o convulsiones y trastornos del estado de conciencia; mientras que en la población adulta se presento malestar general, artralgias, mialgias, acompañadas de fiebre, dificultad respiratoria y exacerbación de la enfermedad respiratoria de base, estos fueron datos característicos observados por el personal de salud (médicos y enfermeras).

El personal de enfermería del INER, adscrito a urgencias fue el primero en hacer frente a esta contingencia de salud, por lo que fue necesaria la organización y coordinación acertada de las acciones implementadas para la atención, manejo y control de la epidemia. Una de ellas fue la participación en la toma de muestra nasofaringea, la cual fue enviada al laboratorio de microbiología del Instituto, para su proceso y análisis. 
Enfermería representa el $30 \%$ de la fuerza laboral de trabajo más importante en el Instituto, ya que participa en el cuidado directo del paciente con afección respiratoria. Durante la contingencia de salud, el personal de enfermería una vez más demostró su profesionalismo, responsabilidad, y compromiso social, al participar activa y decididamente en la atención del paciente con sospecha y/o confirmación de influenza, demostrando que con organización, coordinación de las acciones implementadas y el trabajo en equipo se logra el éxito.

\section{Medidas implementadas durante la Contingencia de Salud en el INER}

El Instituto Nacional de Enfermedades Respiratorias, consciente de su papel normativo en salud respiratoria y ante la amenaza de una posible pandemia de influenza, tomo medidas de prevención, información y organización en coordinación con personal de salud, con la finalidad de establecer una diferencia en el manejo de la situación sanitaria durante la contingencia, cuyo objetivo fue la creación de un cerco sanitario dentro del instituto y evitar la propagación y posibles contagios dentro y fuera de la institución.

El Objetivo de este artículo es describir desde la óptica laboral, la participación, organización y coordinación del área de enfermería, ante la epidemia de Influenza A (H1N1) que se presento durante el periodo de marzo-mayo del 2009 en el INER y las actividades que se realizaron en las áreas de concentración, para dar respuesta a la alerta epidemiológica. Cuyas actividades fueron las siguientes:

\section{DEPARTAMENTO DE ENFERMERÍA}

1. Reunión extraordinaria con jefes de área, y supervisoras del Departamento de Enfermería, con el objetivo de informar sobre la emergencia de salud, y elaborar plan de acción para enfermería, estableciendo prioridades, para responder a las exigencias de la alerta epidemiológica.

- Verificar cantidad existente de insumos, en Almacén de Farmacia: mascarilla de alta eficiencia y mascarilla quirúrgica, googles, guantes y bata desechable, así como de Uniformes quirúrgicos de algodón en Almacén General.

- Solicitud extraordinaria de insumos al Departamento de Adquisiciones: Mascarilla N95 (15.000), batas desechables (10.000), googles (2.500) y uniformes quirúrgicos de algodón (200).
- Intensificación de la campaña de vacunación contra influenza a todo el personal de salud del instituto, implementándose horarios vespertino, nocturno, turno especial diurno y nocturno. Actividad realizada por el Departamento de enfermería.

- Supervisión extraordinaria de las áreas físicas designadas para la concentración de pacientes, con sospecha y/o confirmación de influenza, para la detección oportuna de necesidades, dotación suficiente de material y equipo para la prevención, manejo y control de riesgos al paciente, personal de salud y equipo multidisciplinario.

- Coordinación con el Comité de Control de infecciones en el reforzamiento de medidas de prevención (lavado de manos y dotación suficiente de gel alcohol).

- Supervisión estricta del manejo de la técnica de aislamiento respiratorio por el personal de salud y equipo multidisciplinario, durante la atención al paciente.

- Registro estadístico de casos sospechosos y/o confirmados de influenza, así como de familiares y contactos.

- Solicitud de apoyo al comité de Bioseguridad Institucional, para el reforzamiento del uso correcto del EPP al personal de enfermería.

2. Dotación de Equipo de Protección Personal (EEP) completo a enfermería, en las áreas de concentración para la atención de pacientes con Influenza: mascariIla N95, bata desechable, googles, guantes, uniforme quirúrgico, gorro.

- Dotación de material suficiente para la atención del paciente. $^{3}$

3. Programación de sesiones informativas sobre influenza estacional, dirigido a todo el personal de enfermería y personas interesadas en el tema, impartida por la Lic. Enf. Lilia Damián Yañez, Jefe del Departamento de Enfermería, en las siguientes fechas y horarios:

- 17 de abril 17:00 hrs. y 22:00 hrs.

- 18 de abril 10:00 hrs. y 22:00 hrs.

- 20 de abril 9:00 hrs.

- 21 de abril 22:00 hrs.

Lugar: Auditorio Donato G. Alarcón.

4. El día 23 de abril el Secretario de Salud José Ángel Córdova Villalobos, emitió el mensaje del inicio de la Contingencia de Salud ante la epidemia, hasta ese momento considerada como Influenza estacional; sin embargo, después de haber enviado muestras 
en coordinación con el INDRE (Instituto Nacional De Referencia Epidemiológica) a laboratorios de Canadá y Estados Unidos, confirmaron que se trataba de un nuevo virus de la influenza. El INER al ser un hospital de especialidad y referencia, concentró a un número considerable de pacientes con dicha enfermedad, siendo necesaria la reconversión hospitalaria, en la cual participo activamente el personal de enfermería, esta incluye las siguientes medidas:

- Suspensión de consultas de primera vez y subsecuentes.

- Disminuir Estancia de familiares en sala de espera.

- Asignación de 6 consultorios para atención de pacientes con sospecha de influenza.

- Triage en la atención de pacientes en consulta de Urgencias (cuadro 2).

- Implementación de base de datos electrónica para pacientes, familiares y contactos (información al servicio de Epidemiología clínica).

- Restringir acceso al servicio de urgencias respiratorias a personal no autorizado.

- Habilitación de áreas físicas en la Unidad de Urgencias Respiratorias para la atención de pacientes críticos (área de choque y recuperación), al rebasar la capacidad de la unidad.

- Reforzamiento de las medidas de prevención y EPP en la atención del paciente.

- Agilización de trámites administrativos para la hospitalización oportuna del paciente.

- Suspensión de cirugías programadas de ORL.

- Asignación de 2 servicios clínicos para la concentración y atención de pacientes con sospecha de influenza con capacidad de 30 camas.

- Traslado de pacientes en estado crítico de las unidades de terapia intensiva e intermedia, con diagnóstico de EPOC, Asma, entre otros, a la unidad de terapia intensiva del INCan (Instituto Nacional de Cancerología).

- Cierre temporal del servicio de ORL y reubicación del personal médico y de enfermería a los servicios de concentración.

- Reestructuración de la visita familiar (horario, número de visitantes). Lineamientos específicos para la visita de pacientes con sospecha de influenza (reforzamiento en el lavado de manos y uso de gel alcohol, uso de bata clínica y mascarilla quirúrgica). visita solo a través del cristal de la habitación.
5. Se implemento rol de guardias semanal para jefes de área del Depto. de Enfermería, con el objetivo de coordinar las actividades del personal operativo adscrito a los servicios de concentración, proporcionar EPP y material necesario para la atención oportuna del paciente (mascarillas N95, guantes, batas desechables). Detectar y resolver problemas emergentes durante la contingencia.

6. Reforzamiento de la plantilla del personal operativo de enfermería en los servicios de concentración, con el objetivo de garantizar la atención de calidad al paciente durante la contingencia y disminuir riesgos laborales por sobre carga de trabajo.

7. Asignación de personal supervisor a las áreas de concentración como apoyo a Jefe de servicio, para la gestión administrativa de material y equipo biomédico adicional

8. Integración de Enfermería al comité de Influenza institucional.

9. Solicitud de tratamiento profiláctico a la Dirección General para personal de enfermería en atención directa al paciente con sospecha y/o confirmación de influenza.

10. Emisión de lineamientos específicos durante la Contingencia, para el manejo del paciente:

- Reforzamiento de medidas de prevención (lavado de manos y uso de gel alcohol, antes y después de la atención al paciente).

- Uso de uniforme quirúrgico completo.

- EPP estricto (mascarilla de alta eficiencia N95, N99, googles, bata clínica y bata desechable no estéril, gorro).

- Manejo de técnica de aislamiento respiratorio.

- Manejo estricto de RPBI.

- Baño de regadera al termino de la jornada laboral.

11. Gestión administrativa para la contratación emergente de personal de enfermería para cubrir necesidades en la atención del paciente, por sobre demanda de servicios de salud durante la contingencia en el INER.

12. Apoyo al personal de enfermería que presento síntomas de influenza, así como a familiares y contactos: canalización a consultorio de atención médica, para trabajadores del Instituto y dotación de antivirales.

13. Otorgamiento de días de descanso a las personas en periodo de gestación o lactancia y personal que presento síntomas de influenza, de acuerdo con lo emitido por la Secretaria de Salud. 


\section{UNIDAD DE URGENCIAS}

El trabajo desarrollado en el área de Urgencias, es efectuado por un grupo de profesionales conformado mayoritariamente por enfermeras/os, quienes requieren de conocimientos especializados para manejo avanzado de patologías y de situaciones de urgencia, que pueden marcar la diferencia entre la vida y la muerte del paciente; desde el punto de vista personal, implica un compromiso constante de superación y entrega.

El personal de enfermería del Instituto adscrito a este servicio; como se ha mencionado, fue el primer contacto en hacer frente a la emergencia de salud, por lo que fue necesario la organización y coordinación acertada de las acciones implementadas para la atención, manejo y control de la epidemia, las cuales iniciaron con el Triage en la consulta de Urgencias (cuadro 2). La participación de la enfermera/o en esta clasificación fue trascendental, al valorar el nivel de gravedad del paciente, aplicar los cuidados específicos y el traslado inmediato al servicio de concentración, para continuar con su tratamiento, minimizando riesgos y favoreciendo la pronta recuperación. ${ }^{3,4}$ Proceso (Cuadro 1).

\section{Cuadro 1. Proceso de atención en consulta de urgencias.}

\begin{tabular}{|c|c|c|}
\hline Secuencia de Etapas & Actividad & Responsable \\
\hline 1. Medidas de Prevención & 1.1 Lavado de manos y uso de gel alcohol. & Médico y Enfermera \\
\hline $\begin{array}{l}\text { 2. Equipo de Protección Personal } \\
\text { (EPP). }\end{array}$ & $\begin{array}{l}\text { 2.1 Uso de Mascarilla N95 o N99, googles, gorro, guantes, } \\
\text { uso de uniforme quirúrgico. }\end{array}$ & Medico y Enfermera \\
\hline 3. Registro del paciente & $\begin{array}{l}\text { 3.1 Registra datos personales del paciente en bitácora } \\
\text { correspondiente. }\end{array}$ & Enfermera \\
\hline 4. Toma de signos vitales & $\begin{array}{l}\text { 4.1 Realiza toma de T/A, FC, FR, Temperatura, anotando } \\
\text { datos en ficha del paciente. }\end{array}$ & Enfermera \\
\hline $\begin{array}{l}\text { 5. Instalación de monitoreo } \\
\text { de oximetría de pulso. }\end{array}$ & $\begin{array}{l}\text { 5.1 Prepara equipo y coloca dedal al paciente, registrando } \\
\text { resultado en ficha del paciente. }\end{array}$ & Enfermera \\
\hline $\begin{array}{l}\text { 6. Registro de saturación de oxigeno }>70 \% \text {, } \\
\text { sin compromiso respiratorio. } \\
\text { - Inicio de tratamiento antiviral. }\end{array}$ & $\begin{array}{l}\text { 6.1 Solicita placa AP de tórax urgente y envía paciente a RX. } \\
\text { 6.2 Realiza procedimiento para la toma de muestra } \\
\text { nasofaringea con trampa. } \\
\text { 6.3 Prescribe tratamiento antiviral (Oseltamivir) a paciente } \\
\text { con sintomatología sugestiva de influenza. } \\
\text { 6.4 Administra antiviral. }\end{array}$ & $\begin{array}{l}\text { Médico } \\
\text { Enfermera } \\
\text { Médico } \\
\text { Enfermera }\end{array}$ \\
\hline $\begin{array}{l}\text { 7. Alta del paciente. } \\
\text { - Medidas de prevención domiciliaria. }\end{array}$ & $\begin{array}{l}\text { 7.1 Proporciona información sobre signos y síntomas } \\
\text { de alarma. } \\
\text { 7.2 lavados de manos, uso de gel alcohol, uso de mascarilla } \\
\text { quirúrgica, medidas higiénico dietéticas. }\end{array}$ & $\begin{array}{l}\text { Médico } \\
\text { Enfermera }\end{array}$ \\
\hline $\begin{array}{l}\text { 8. Saturación de oxigeno }<70 \% \text {, } \\
\text { con compromiso respiratorio. }\end{array}$ & $\begin{array}{l}\text { 8.1 Aviso a jefe de servicio de urgencias, sobre el ingreso } \\
\text { directo del paciente grave. }\end{array}$ & Enfermera \\
\hline 9. Paso directo a urgencias observación. & $\begin{array}{l}\text { 9.1 Solicita apoyo de camillero y traslada a paciente. } \\
\text { Colocando mascarilla quirúrgica. }\end{array}$ & Enfermera y Camillero \\
\hline
\end{tabular}


Cuadro 2. Triage ante un caso probable

y/o confirmado de Influenza. Consulta.

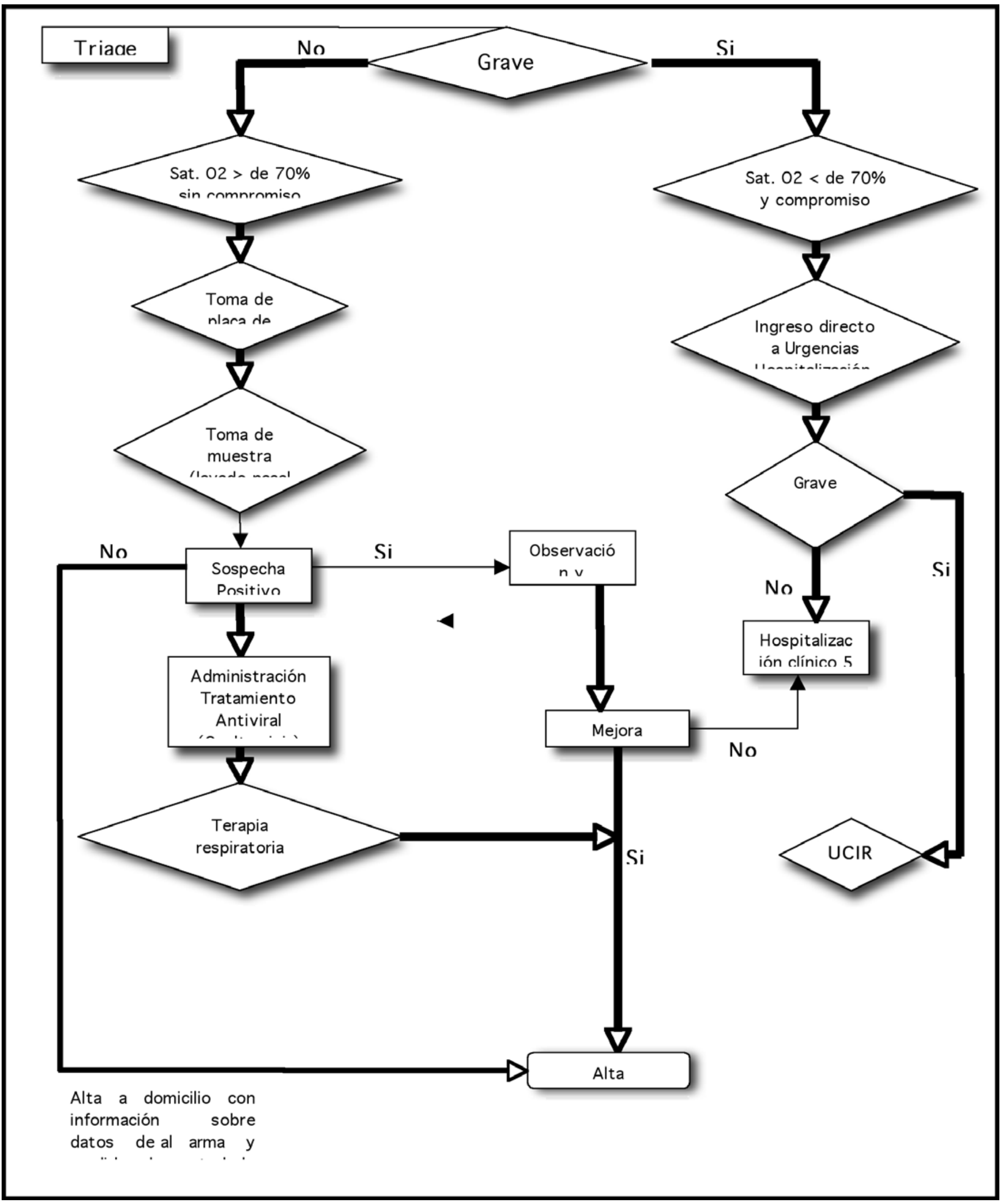




\section{URGENCIAS ATENCIÓN DEL PACIENTE EN URGENCIAS HOSPITALIZACIÓN.}

1. Uso de medidas de prevención y EPP para la atención del paciente

2. Registro del paciente.

3. Manejo de la vía aérea:

- Aporte de O2 al 100\% por mascarilla con bolsa reservoria

- Canalización de acceso venoso central y/o periférico.

- Monitorización cardiaca no invasiva y Sat. O2

- Aislamiento respiratorio.

4. En pacientes con incremento del trabajo respiratorio, inicio de ventilación mecánica no invasiva (BPAP), al no ser suficiente el aporte de $\mathrm{O} 2$ con esta modalidad, inicio de ventilación mecánica invasiva.

5. Toma de muestra (lavado nasal y bronquial con trampa) por enfermería.

6. Se brindan cuidados al paciente en estado crítico con apoyo ventilatorio, hemodinamicamente inestable.

- Durante la estancia hospitalaria, se observo que los pacientes presentaban fiebre $>$ de $40^{\circ} \mathrm{C}$ sin respuesta a medios físicos o químicos, abundantes secreciones de características asalmoneladas, acidosis metabólica, falla renal súbita, sin respuesta favorable a tratamiento.

- Todos los pacientes graves que requirieron de AMV presentaban signos, síntomas y complicaciones anteriormente mencionados con deterioro rápido y progresivo que los llevaba a la muerte.

7. Traslado del paciente a la Unidad de Cuidados Intensivos Respiratorios (UCIR) o servicio clínico 5.

\section{Unidades Terapia Intensiva e Intermedia y servicio clínico 5}

La atención del paciente crítico requiere una acción eficaz y rápida, por personal altamente capacitado para establecer condiciones diferenciadas que propicien su recuperación, características que el personal de enfermería del INER cumple, para brindar cuidados integrales al paciente con sospecha y/o confirmación de influenza, ingresado a las Unidades de Terapia Intensiva e Intermedia y clínico 5 ,

Durante la contingencia de salud se reforzaron algunos lineamientos, medidas de prevención y protección ya establecidas en las unidades, con el objetivo de minimizar los riesgos para el personal, paciente y familiares.
- Reforzamiento en el uso de medidas de prevención (lavado de manos y uso de gel alcohol.

- Uso de EPP completo.

- Uso de uniforme quirúrgico, otorgado por la CEyE.

- Aislamiento respiratorio para todos los pacientes.

- Manejo estricto de RPBI (uso de bolsa roja en toda la unidad).

- Restricción de acceso a personal no autorizado.

- Restricción de visita familiar, de acuerdo a lo establecido en la reconversión hospitalaria.

- Restricción del personal de salud en atención directa del paciente con sospecha y/o confirmación de influenza, a deambular en áreas comunes con uniforme quirúrgico.

- Baño de regadera al final de la jornada laboral. ${ }^{5}$

\section{RECOMENDACIONES}

La presencia de la epidemia de influenza en la primavera del 2009, se proyecto como un desafió para el país así

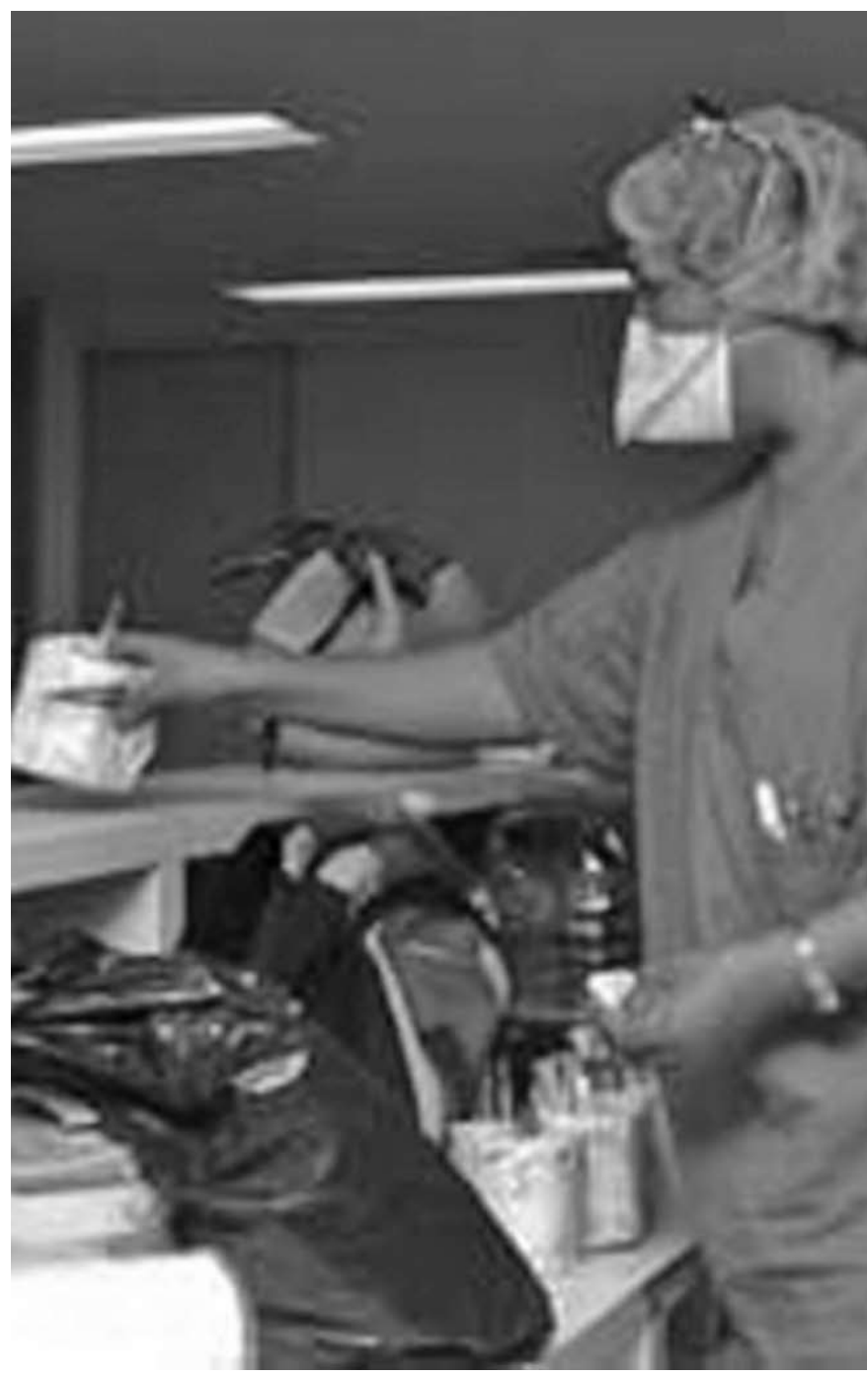


como para todas y cada una de las instituciones de salud que dieron atención directa al paciente sospechoso y/o confirmado de Influenza A H1N1, el INER no fue la excepción pues esta enfermedad se presento de manera inesperada, rebasando la capacidad de atención médica en el instituto, esta situación genero como consecuencia la gestión emergente de recursos humanos y materiales, dando inicio con la organización de un plan emergente mediante la formación del comité de influenza Institucional, que se responsabilizo de los acuerdos y acciones para solucionar las necesidades inmediatas que surgieron durante la evolución de la epidemia.

El Instituto Nacional de Enfermedades Respiratorias al ser una entidad normativa en salud respiratoria a nivel nacional y siguiendo los lineamientos establecidos internacionalmente por la OMS, actuó en respuesta a la alerta sanitaria a traves de este comité aplicando las medidas y acciones estrictas de seguridad necesarias para limitar la propagación del virus, por lo que ante la experiencia vivida se recomienda establecer de forma permanente las acciones descritas en este articulo para el profesional de Enfermería.

- Formalizar el plan de contingencia Institucional especifico de Enfermería.

- La motivación y la actitud del personal de Enfermería durante la ejecución de sus actividades, se puede ver afectada en su desempeño al sentir miedo, angustia e incertidumbre ante lo desconocido, por lo que la información veraz y oportuna juega un papel fundamental en el cuidado integral del paciente.

- Evaluar crónicamente la planeación y ejecución de dicho plan para lograr mejoras continuas a través de la actualización, que nos permitan ser competentes en futuras contingencias.

- Resaltar la importancia de mantener el uso de medidas universales de protección estándar no solo durante la presencia de contingencias, sino que debe ser una acción permanente durante el desarrollo de nuestra práctica profesional.

- Reconocer y aplaudir el trabajo realizado por el personal de enfermería y todo el equipo multidisciplinario que intervino durante el plan emergente.

- Nunca dejar de lado, que la práctica de enfermería se rige por un enfoque Holístico por ello, no debemos olvidar el apoyo comunitario para las familias afectadas por tal padecimiento.
- Por ultimo no olvidemos que el objetivo de nuestra práctica profesional es y será por siempre salvaguardar la salud integral de todo ser humano.

Con todo ello nos queda claro que el Instituto Nacional de Enfermedades Respiratorias, consciente de su papel normativo en salud respiratoria y ante la amenaza de una posible pandemia de influenza, tomo medidas preventivas, de información y organización del personal de salud, con la finalidad de establecer una diferencia en el manejo de la situación sanitaria durante la contingencia y ser el pionero en la creación de un Plan de Contingencia, para la atención del paciente, o bien como la base principal de planeaciones estratégicas para futuras contingencias. Sin dejar de mencionar que gracias a la valiosa cooperación del cuerpo enfermero, todo ello no hubiera sido posible.

\section{REFERENCIAS BIBLIOGRÁFICAS}

1. Cárdenas de la Peña E. Del Sanatorio Huipulco, al Instituto Nacional de Enfermedades Respiratoria. Cincuenta años. México, 1986.

2. Secretaría de Salud. Acciones básicas ante la intensificación de la transmisión de influenza.- Boletín Influenza.pdf 2. México. 2009

3. Manual de procedimientos técnicos del departamento de enfermería INER 2009

4. Smith NM, S. BJ, Shay DK, Uyeki TM, Cox NJ and A. SR. Prevention and Control of Influenza. Recommendations of the Advisory Committee on Immunization Practices (ACIP). MMWR Recomm Rep 2006;55:1-42.

5.Secretaría de Salud. Plan Nacional para la atención de la Pandemia Aviar. México, 2007.

\section{DIRECCIÓN PARA CORRESPONDENCIA}

Lic. Ma. Teresa Pérez López: lutere21@hotmail.com

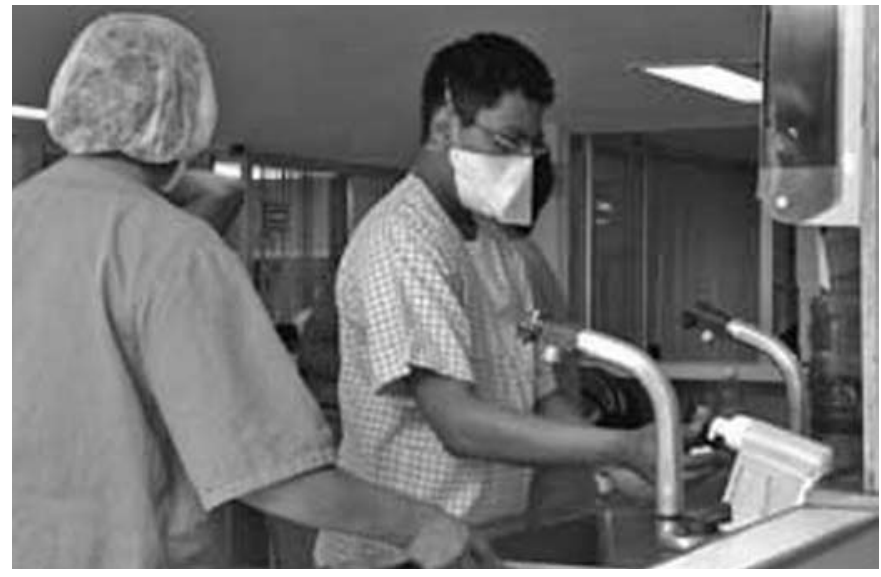

